\title{
REVISÃO DE LITERATURA Programas metacognitivos com enfoque em cognição social na reabilitação da esquizofrenia: uma revisão sistemática
}

\author{
Metacognitive programs focusing social cognition for the \\ rehabilitation of schizophrenia: a systematic review
}

Hélio Anderson Tonelli', Fernanda Liboni', Diego Augusto Nesi Cavicchioli²

\section{RESUMO}

Objetivo: Revisar sistematicamente a literatura sobre programas psicoterapêuticos para a esquizofrenia com enfoque em CS, cujos métodos envolvam estratégias metacognitivas. Métodos: Foi realizada uma busca na base MedLine, por trabalhos publicados em inglês ou português utilizando a frase "Social cognition" AND "Schizophrenia" [Mesh] AND "Psychotherapy" [Mesh] e os limitadores "Humans", "Clinical Trial", "Meta-Analysis" e "Randomized Controlled Trial". Adicionalmente, foram elaborados critérios de inclusão para selecionar os trabalhos com abordagem metacognitiva. Resultados: Dezessete estudos foram selecionados, abrangendo essencialmente programas de reconhecimento do afeto facial e de emoções, Teoria

\section{Palavras-chave}

Esquizofrenia, cognição social, psicoterapia, sociobiologia.

\section{Keywords}

Schizophrenia, social cognition, psychotherapy, sociobiology. da Mente (ToM), imitação e tomada de perspectiva em situações sociais. Conclusão: A maior parte dos estudos mostrou que seus programas são eficazes para melhorar medidas de psicopatologia, CS e funcionamento social. Futuras pesquisas deverão esclarecer sobre segurança, especificidade e durabilidade dessas intervenções.

\section{ABSTRACT}

Objective: To review systematically the literature on psychotherapeutic programs focusing on SC, designed to schizophrenia, which methods include metacognitive strategies. Methods: A search on MedLine base for papers published in English or Portuguese has been performed, using the phrase "Social cognition" AND "Schizophrenia" [Mesh] AND "Psychotherapy" [Mesh] and the limits "Humans", "Clinical Trial", "Meta-Analysis" and "Randomized Controlled Trial". Additionally, inclusion criteria have been formulated in order to select papers with metacognitive approach. Results: Seventeen articles have been selected, which comprised essentially facial emotion recognition, emotion recognition, Theory of Mind (ToM), imitation and perspective taking in social situations. Conclusion: The great majority of papers have shown that their programs are effective in improving measures of psychopathology, SC and social functioning. Future research might clarify about safety, specificity and durability of such interventions.

1 Instituto de Psiquiatria do Paraná.

2 Universidade Estadual de Londrina (UEL).

Recebido em

12/11/2012

Aprovado em

$3 / 2 / 2013$

Endereço para correspondência: Hélio Anderson Tonelli

Av. Cândido de Abreu, 526, cj. 311, Torre B

80530-000 - Curitiba, PR, Brasil

Email: hatonelli@gmail.com 


\section{INTRODUÇÃO}

Por se tratar de uma doença crônica e potencialmente incapacitante, a esquizofrenia é considerada um sério problema de saúde pública, para o qual estratégias terapêuticas multidimensionais devem, idealmente, ser desenvolvidas.

Abordagens psicoterapêuticas individuais ou grupais são coadjuvantes do tratamento farmacológico e podem ser empregadas de forma fácil, tanto em pacientes ambulatoriais quanto em internados.

Os principais alvos terapêuticos dessas abordagens costumam ser as dificuldades interpessoais e sintomas resistentes à medicação.

Circuitos cerebrais especificamente relacionados ao processamento da informação do ambiente social - ou cognição social (CS) - estão disfuncionais na esquizofrenia, gerando sintomas que poderiam ser entendidos como reações "para mais" ou "para menos" a esse ambiente (respectivamente, sintomas paranoides e afastamento social)'.

Por auxiliar na compreensão da origem dos sintomas da esquizofrenia, o estudo da CS pode ser útil para o desenvolvimento de estratégias psicoterapêuticas a serem empregadas com a farmacoterapia, melhorando a evolução do transtorno em médio e longo prazo.

A maior parte das técnicas de psicoterapia e reabilitação desenhadas para a esquizofrenia dirige-se tanto à melhora cognitiva geral quanto da interação social, e as mais estudadas são as psicoterapias de remediação cognitiva e o treino de habilidades sociais. Embora úteis, esses programas não levam em conta as características qualitativas dos estímulos cognitivos sociais², tendendo a considerar as distorções apresentadas pelos pacientes em suas interpretações do ambiente social mais como uma consequência de prejuízos cognitivos gerais, como déficits atencionais e de função executiva. Dessa forma, essas intervenções têm uma abordagem baseada nos sintomas, ou, segundo Aghotor et al. ${ }^{3}$, do tipo "porta da frente", por não considerarem a origem cognitiva social dos sintomas. Assim, o foco das psicoterapias cognitivas para a esquizofrenia seria a modificação de crenças e esquemas mal-adaptativos decorrentes de déficits cognitivos ${ }^{4}$, e não da cognição propriamente dita. Programas com foco na cognição baseiam-se na melhora de parâmetros cognitivos gerais (memória, aprendizado, atenção e função executiva) ou sociais (reconhecimento de emoções, capacidade de inferência de estados mentais em outros indivíduos e estilos atribucionais) e seriam mais adequadamente designados como "treino cognitivo" e considerados estratégias do tipo "porta dos fundos".

Abordagens metacognitivas, por sua vez, são desenvolvidas para focar vieses cognitivos subjacentes aos sintomas esquizofrênicos e promover alguma reflexão sobre os próprios processos cognitivos. O termo "metacognição" foi utilizado pela primeira vez em 1976 por Flavell', que o definiu como "aquilo que se refere ao conhecimento que alguém tenha a respeito de seus próprios processos cognitivos, ou a qualquer coisa que a estes processos se relacionem (...)". Metacognição, portanto, define a cognição acerca de cognição, isto é, aquilo que sabemos sobre e reconhecemos em nossa própria cognição.

Programas metacognitivos com enfoque em CS empregam diversas tarefas que têm em comum a avaliação supervisionada, o estímulo à reflexão e o aprendizado baseados na observação de como a mente interpreta e reage aos mais diversos estímulos sociais. Tais técnicas podem auxiliar na correção de erros na interpretação desses estímulos, levando a comportamentos mais adaptativos e à melhora nos relacionamentos interpessoais.

Este manuscrito tem como objetivo revisar sistemática e criticamente a literatura sobre programas psicoterapêuticos para a esquizofrenia que se baseiem em CS (ou incluam em algum de seus subprogramas), cujo método envolva estratégias metacognitivas, procurando por ensaios clínicos que comparem esses programas a outros métodos terapêuticos não farmacológicos já tradicionalmente utilizados em settings ambulatoriais ou hospitalares.

\section{MÉTODOS}

Em uma primeira etapa da preparação deste manuscrito, foi realizada uma busca na base de dados MedLine, por trabaIhos publicados em português ou inglês, no período compreendido entre 1/1/1990 e 1/8/2011, utilizando a frase de busca "Social cognition" AND "Schizophrenia" [Mesh]) AND "Psychotherapy" [Mesh] e os limitadores "Humans", "Clinical Trial", "Meta-Analysis" e "Randomized Controlled Trial". Essa busca tinha por finalidade encontrar estudos sobre intervenções psicoterápicas utilizando conceitos sociocognitivos e resultou em 11 artigos ${ }^{2,6-15}$, dos quais um foi excluído por não se tratar de um ensaio clínico 7 . Outros 10 manuscritos $3,16-24$ foram selecionados a partir da leitura dos artigos acima, pois pareciam preencher os critérios de inclusão do tema a ser discutido. Após a leitura deles, dois foram descartados por não se tratarem de ensaios clínicos ${ }^{22,24}$ e outro ${ }^{23}$, por não envolver diretamente CS.

Em uma segunda etapa, foram selecionados os artigos que estudaram estratégias psicoterapêuticas metacognitivas. Uma vez que nem todos os trabalhos selecionados explicitavam isso, procurou-se estabelecer um critério objetivo com o qual fosse possível afirmar que estratégias metacognitivas de tratamento haviam sido utilizadas. Assim, a alusão à abordagem do tipo "porta de trás" foi escolhida na elaboração desses critérios: segundo ela, o principal objetivo deve ser a mudança da "infraestrutura cognitiva" por meio do aumento da atenção dos pacientes à própria cognição. De acordo com essa perspectiva, optou-se por definir, se não 
mencionado no manuscrito, que uma estratégia metacognitiva fora adotada quando o método incluía o favorecimento da conscientização dos pacientes acerca de seus déficits por meio do estímulo à observação, avaliação e/ou simulação e correção dos próprios processos cognitivos sociais ${ }^{3}$. Segundo esses critérios, todos os 17 artigos inicialmente selecionados puderam ser incluídos.

\section{RESULTADOS}

Dos 17 artigos incluídos, 14 são estudos controlados s,6,8-21 $^{3}$ três, não controlados 2,12,17. As principais características desses estudos e seus principais achados estão sumarizados na tabela 1.

De forma geral, os estudos incluídos compararam programas realizados em grupo ou individualmente (os programas metacognitivos avaliados estão resumidos na tabela 2) com outros tipos de intervenção-controle, como grupo de discussão de jornais ${ }^{3}$, terapia de solução de problemas ${ }^{6}$; "estratégias terapêuticas usuais", incluindo manejo de medicação, psicoterapia suportiva, psicoeducação e terapia ocupacional 2,14,18. psicoterapia suportiva9,13,15; seguimento medicamentoso isolado $^{8}$; treino de habilidades sociais ${ }^{19}$ e psicoterapia de remediação cognitiva ${ }^{18}$. Um estudo examinou os efeitos de sua intervenção sobre a psicopatologia ou cognição em portadores de esquizofrenia e comparou-os com controles históricos ${ }^{17}$.

Três programas foram estudados em mais de uma publicação: Cognitive Enhancement Therapy (CET) ${ }^{9,12,13,15}$, Social Cognition Interaction Training (SCIT) 2,11,19 e Training of Affect Recognition (TAR) ${ }^{17,18}$.

Os programas estudados abrangeram várias abordagens, baseadas nas principais linhas de estudo de CS (Tabela 2), incluindo tarefas de reconhecimento e percepção de emoções, processamento cognitivo "teoria da mente" (ou "ToM", associado à habilidade de inferência de estados mentais em terceiros), atribuição e julgamento de situações sociais, tomada de perspectiva, apreciação, avaliação e imitação de ações em contextos sociais.

Tabela 1. Principais características e achados dos trabalhos selecionados

\begin{tabular}{|c|c|c|c|}
\hline Estudos controlados & Número de participantes & Principais características do estudo & Principais achados \\
\hline Penn e Combs, $2000^{16}$ & $\begin{array}{l}40 \text { pacientes internados ( } 23 \text { homens e } 17 \text { mulheres) } \\
\text { com diagnósticos de esquizofrenia ou transtorno } \\
\text { esquizoafetivo, de acordo com a Entrevista } \\
\text { Clínica Estruturada para DSM-IV, foram alocados } \\
\text { aleatoriamente para um dos quatro grupos da } \\
\text { pesquisa }\end{array}$ & $\begin{array}{l}\text { Compararam o impacto da imitação de expressões } \\
\text { faciais sobre o reconhecimento de emoções com } 0 \\
\text { obtido via reforço monetário. Os autores compararam } \\
\text { quatro grupos em relação ao seu desempenho na } \\
\text { identificação do afeto facial e na generalização do } \\
\text { reconhecimento de afeto. Os grupos receberam } \\
\text { abordagens distintas quanto à execução das tarefas: } \\
\text { treino simples, treino com reforço financeiro (a cada } \\
\text { acerto o indivíduo recebia imediatamente } \$ 0,10) \text {, } \\
\text { treino de imitação das emoções mostradas (ou } \\
\text { feedback facial) e treino combinado; feedback facial e }\end{array}$ & $\begin{array}{l}\text { Os grupos "reforço", "feedback facial" e } \\
\text { "combinação" melhoraram significativamente seu } \\
\text { desempenho no teste de identificação do afeto } \\
\text { facial em relação ao treino simples, isto é, houve } \\
\text { ampliação da capacidade de identificar vários tipos } \\
\text { distintos de afetos observados em fotos de faces. } \\
\text { Todavia, houve limitadas evidências de que os } \\
\text { efeitos das intervenções se generalizavam para a } \\
\text { discriminação do afeto facial, isto é, a habilidade } \\
\text { de perceber se duas faces distintas mostradas } \\
\text { sequencialmente exibem a mesma emoção }\end{array}$ \\
\hline
\end{tabular}

Hogarty et al., 2004 120 pacientes ambulatoriais satisfazendo critérios diagnósticos do DSM-II-R ou do DSM-IV para esquizofrenia ou transtorno esquizoafetivo $55 \%$ homens e $41 \%$ mulheres) foram randomizados para Cognitive Enhancement Therapy (CET) ou controle

Roncone et al., 200421 20 portadores de esquizofrenia residual, de acordo com critérios do DSM-IV (13 homens e 7 mulheres), aleatoriamente alocados para Instrumental Enrichment Programme (IEP) ou controle (tratamento medicamentoso e psicoterapia de apoio)

Wölwer et al., 2005 ${ }^{18} \quad 77$ pacientes ambulatoriais com diagnóstico de esquizofrenia de acordo com a CID-10 (60 homens e 17 mulheres), alocados aleatoriamente para os grupos Training of Affect Recognition (TAR); Cognitive Remediation Training (CRT) e tratamento usual

Choi e Kwon, 2006 34 pacientes ambulatoriais diagnosticados com esquizofrenia ou transtorno esquizoafetivo de acordo com DSM-IV (19 homens e 15 mulheres) foram randomicamente alocados para receberem Social Cognitive Enhancement Training (SCET) ou tratamento convencional
Estudaram o efeito do CET sobre a cognição de portadores de esquizofrenia ou transtorno esquizoafetivo. 0 CET foi comparado a técnicas psicoterápicas suportivas ao longo de dois anos. Foram realizadas avaliações cognitivas e psicopatológicas na baseline e aos 12 e 24 meses

Estudaram a eficácia do IEP em uma população de esquizofrênicos com déficits ToM, comparando este programa com um grupo controle, durante seis meses

Compararam o TAR com duas outras estratégias, CRT e tratamento usual (que não incluía treino cognitivo algum), a fim de observar seus efeitos sobre o reconhecimento do afeto facial

Avaliaram o impacto do SCET sobre a avaliação de contexto social em esquizofrênicos. 0 SCET foi comparado a tratamento convencional, caracterizado por atividades de otimização da aderência à medicação e do funcionamento social e ocupacional. 0 s autores utilizaram instrumentos para aferição de CS antes e após o tratamento, que foi realizado por seis meses
Aos 12 meses foi observado um efeito robusto do CET em domínios neurocognitivos, com efeitos marginais sobre a cognição social. No entanto, a partir dos 24 meses houve melhoras significativas no grupo CET em todas as medidas comportamentais e de cognição, exceto em sintomas residuais

Houve melhora estatisticamente significativa do grupo IEP em diversos domínios cognitivos sociais, como habilidades ToM e de reconhecimento de emoções, principalmente tristeza e medo

Os pacientes recebendo TAR apresentaram melhora significativa no reconhecimento do afeto facial em relação aos outros grupos. 0 grupo CRT, de tratamento ativo, não modificou o desempenho sobre o reconhecimento de emoções, mas apresentou melhora na memória verbal

Neste trabalho, o SCET melhorou déficits atencionais, de memória e de função executiva em esquizofrênicos em relação ao tratamento convencional, mas não houve generalização desses efeitos no funcionamento social. Além disso, houve fracas evidências de que o SCET tenha efeito sobre 0 reconhecimento de emoções 


\begin{tabular}{|c|c|c|}
\hline Estudos controlados & Número de participantes & Principais características do estudo \\
\hline Combs et al., $2007^{19}$ & $\begin{array}{l}28 \text { pacientes ambulatoriais de um serviço de } \\
\text { psiquiatria forense ( } 21 \text { homens e } 7 \text { mulheres), } \\
\text { com diagnóstico de transtorno do espectro da } \\
\text { esquizofrenia de acordo com a Entrevista Clínica } \\
\text { Estruturada para DSM-IV }\end{array}$ & $\begin{array}{l}\text { Compararam o Social Cognition and Interaction } \\
\text { Training (SCIT) a uma estratégia de treino de } \\
\text { habilidades sociais em indivíduos com transtornos } \\
\text { do espectro da esquizofrenia ao longo de } 18 \\
\text { semanas }\end{array}$ \\
\hline
\end{tabular}

Eack et al., $2007^{13}$

38 pacientes ambulatoriais com diagnóstico de esquizofrenia ou transtorno esquizoafetivo de acordo com a Entrevista Clínica Estruturada para DSM-IV (26 homens e 12 mulheres), alocados aleatoriamente para CET ou EST [Terapia Suportiva Enriquecida (Enriched Supportive Therapy)]

Combs et al., 2009"

18 pacientes do estudo de Combs et al., $2007^{19}(12$ homens 6 mulheres)

Eack et al., $2009^{\circ}$

Gil Sanz et al., 200910 14 pacientes ambulatoriais diagnosticados de acordo com os critérios da CID-10 (7 homens e 7 mulheres), aleatoriamente alocados para os grupos experimental e controle

Matsui et al., $2009^{8}$

20 pacientes ambulatoriais preenchendo critérios do DSM-IV para esquizofrenia, alocados aleatoriamente para reabilitação cognitiva com ênfase em informação social ou controle
Aghotor et al., $2010^{3} \quad 30$ portadores de transtorno do espectro da esquizofrenia (20 homens e 10 mulheres), de acordo com critérios da (ID-10, foram alocados para Metacognitive Training (MCT) ou grupo controle
Examinaram os efeitos do CET sobre a CS em esquizofrênicos com doença recente. 0 CET foi comparado a 0 EST, caracterizado por estratégias de redução do estresse e psicoeducação, ao longo de um ano

Publicaram dados acerca da estabilidade dos efeitos no grupo SCIT na população anteriormente estudada por Combs et al. $(2007)^{19}$. Além de submeterem essa população a medidas de percepção de emoções e funcionamento social após seis meses, os autores compararam essa população com um grupo de indivíduos de uma comunidade não psiquiátrica

Examinaram os efeitos do CET sobre a CS em esquizofrênicos e portadores de transtorno esquizoafetivo com doença recente, reforçando que podem existir diferenças na capacidade de resposta a métodos psicoterapêuticos em sujeitos com doença recente e tardia graças à preservação da neuroplasticidade naqueles. 0 estudo foi realizado ao longo de dois anos. 0 CET foi comparado à terapia suportiva enriquecida (EST), e os sujeitos experimentais foram avaliados no primeiro e no segundo ano

Examinaram os efeitos da aplicação do Social Cognition Training Program (SCTP), sobre 0 reconhecimento de emoções e percepção social em esquizofrênicos ao longo de 10 semanas

Os autores compararam um programa de reabilitação cognitiva com ênfase em informação social com tratamento convencional (definido como seguimento medicamentoso), ao longo de três meses. Apesar de o programa avaliado não ter um módulo desenhado especificamente para o tratamento cognitivo social, os autores tinham como alvo principal proporcionar um aumento na competência da avaliação de situações sociais, por meio de uma estreita interação pacienteterapeuta, o que, em última análise, favoreceria a criação de novas macroestruturas cognitivas ou representações mentais sobre 0 ambiente social

Compararam a eficácia e a segurança do MCT com um grupo controle ativo sem características psicoterápicas em portadores de transtornos do espectro da esquizofrenia
Principais achados

Além de demonstrar melhora em todos os parâmetros CS, os autores reportaram diminuição de incidentes agressivos no grupo $\mathrm{SCIT}$, argumentando que um bom rendimento cognitivo social permite a configuração de relações interpessoais mais saudáveis, diminuindo o risco de agressão em indivíduos com transtornos do espectro da esquizofrenia. As alteraçōes favoráveis observadas na cognição dos indivíduos recebendo SCIT mantiveram-se mesmo após terem sido estatisticamente controladas com os dados sobre alteraçōes sintomáticas ao longo do tempo

0s indivíduos recebendo CET apresentaram melhora significativa na compreensão e no manejo de emoções, tanto em si mesmos quanto em terceiros; e na habilidade de utilizar emoções para facilitar o pensamento e a tomada de decisões, desenvolvendo a habilidade de compreender 0 significado das emoções em um contexto social. Não houve, contudo, diferenças significativas na competência de perceber acuradamente emoções

0 s resultados foram favoráveis ao $\mathrm{SCIT}$, já que seis meses após o primeiro estudo os escores do grupo SCIT mantiveram-se significantemente maiores do que na baseline, além de não ter havido diferenças entre o desempenho desse grupo e do grupo não clínico

No primeiro ano os indivíduos recebendo CET tiveram melhoras importantes em estilos cognitivos disfuncionais, CS e sintomatologia, em relação ao grupo EST. Após dois anos as diferenças se tornaram altamente significativas favorecendo o CET

0 grupo SCTP mostrou desempenho significativamente superior nas medidas de percepção e interpretação de situações sociais, mas não de reconhecimento de emoções em relação ao grupo controle, que não recebeu treino em percepção social

Os indivíduos recebendo reabilitação cognitiva demonstraram desempenho significativamente maior em medidas de $C S$ em relação ao controle. Tais melhoras foram mantidas por pelo menos três meses após o final da intervenção

0s sujeitos recebendo $\mathrm{MCT}$ apresentaram melhoras tanto em parâmetros psicopatológicos quanto cognitivos, contudo nenhum resultado teve significação estatística 


\begin{tabular}{|c|c|c|c|}
\hline Estudos controlados & Número de participantes & Principais características do estudo & Principais achados \\
\hline Mazza et al., $2010^{6}$ & $\begin{array}{l}32 \text { pacientes ambulatoriais diagnosticados } \\
\text { com esquizofrenia de acordo com a Entrevista } \\
\text { Estruturada do DSM-IV-TR ( } 13 \text { mulheres e } 19 \\
\text { homens), aleatoriamente alocados para receberem } \\
\text { Emotion and ToM Imitation Training (ETIT) ou } \\
\text { Treinamento em Solução de Problemas (TSP) }\end{array}$ & $\begin{array}{l}\text { Compararam o ETIT, baseado na observação de } \\
\text { emoções e na imitação de ações como fontes de } \\
\text { melhora cognitiva social na esquizofrenia, com TSP }\end{array}$ & $\begin{array}{l}0 \text { grupo ETIT apresentou melhor desempenho } \\
\text { em medidas de processamento ToM, no } \\
\text { reconhecimento de determinadas emoções, como } \\
\text { raiva, tristeza e nojo; no desempenho social e em } \\
\text { escores da dimensão positiva medidas pela escala } \\
\text { BPRS em relação ao grupo TSP }\end{array}$ \\
\hline Veltro et al., $2011^{20}$ & $\begin{array}{l}24 \text { pacientes ambulatoriais diagnosticados de } \\
\text { acordo com os critérios do DSM-IV-TR foram } \\
\text { alocados aleatoriamente para Problem Solving } \\
\text { Treatment (PST) ou Cognitive Emotional } \\
\text { Rehabilitation (REC) }\end{array}$ & $\begin{array}{l}\text { Compararam duas estratégias semelhantes em sua } \\
\text { forma, mas distintas em seu foco terapêutico para o } \\
\text { tratamento da esquizofrenia: o PST e a REC. Ambos } \\
\text { utilizam técnicas metacognitivas baseadas em } \\
\text { um trabalho supervisionado; contudo, a REC tem } \\
\text { como principal alvo o reconhecimento de emoções } \\
\text { e o PST, o treino no planejamento de solução de } \\
\text { problemas }\end{array}$ & $\begin{array}{l}\text { Embora os treinos tenham sido diferencialmente } \\
\text { eficientes em seus domínios específicos de ação, } \\
\text { ambos associaram-se a melhoras na psicopatologia } \\
\text { e no funcionamento social }\end{array}$ \\
\hline Fromann et al., $2003^{17}$ & $\begin{array}{l}16 \text { pacientes portadores de esquizofrenia ( } 13 \\
\text { homens e } 3 \text { mulheres) de acordo com o International } \\
\text { Diagnostic Checklist of ICD-10 }\end{array}$ & $\begin{array}{l}\text { Os autores aplicaram o TAR em uma pequena } \\
\text { amostra de esquizofrênicos. Em seguida, } \\
\text { compararam os resultados com dois grupos controle } \\
\text { históricos, compostos de indivíduos saudáveis e de } \\
\text { portadores de esquizofrenia }\end{array}$ & $\begin{array}{l}\text { A maior parte dos pacientes da amostra atual } \\
\text { apresentou melhora significativa na capacidade de } \\
\text { reconhecer afeto facial não atribuível a eventuais } \\
\text { diferenças no estado psicopatológico observadas } \\
\text { antes e após o treino }\end{array}$ \\
\hline Hogarty et al., $2006^{12}$ & 106 pacientes do estudo de Hogarty et al., $2004^{15}$ & $\begin{array}{l}\text { Examinaram se os efeitos observados nos } \\
\text { indivíduos recebendo CET do estudo de Hogarty et } \\
\text { al. (2004) }{ }^{15} \text { eram mantidos após um ano. Os autores } \\
\text { reavaliaram os pacientes daquele estudo um ano } \\
\text { mais tarde, do ponto de vista neuropsicológico e } \\
\text { comportamental }\end{array}$ & $\begin{array}{l}\text { Houve manutenção das melhoras tanto na CS } \\
\text { quanto em outros domínios cognitivos gerais }\end{array}$ \\
\hline Roberts e Penn, $2009^{2}$ & $\begin{array}{l}31 \text { pacientes ambulatoriais com diagnóstico de } \\
\text { transtornos do espectro da esquizofrenia de } \\
\text { acordo com a Entrevista Estruturada para DSM-IV } \\
\text { (13 mulheres e } 18 \text { homens) receberam SCIT ou } \\
\text { "tratamento usual" }\end{array}$ & $\begin{array}{l}\text { Compararam o SCIT com tratamento usual (manejo } \\
\text { de medicação, técnicas de psicoterapia individual } \\
\text { ou grupal, manejo de caso, psicoeducação e terapia } \\
\text { ocupacional) em portadores de transtornos do } \\
\text { espectro da esquizofrenia, ao longo de } 20 \text { semanas }\end{array}$ & $\begin{array}{l}\text { Os indivíduos recebendo SCIT também receberam } \\
\text { o tratamento usual. } 0 \text { grupo SCIT melhorou } \\
\text { significativamente a performance na percepção } \\
\text { de emoções, não havendo efeito significativo } \\
\text { nas medidas ToM nem em vieses atribucionais } \\
\text { agressivos }\end{array}$ \\
\hline
\end{tabular}

Tabela 2. Principais características dos programas estudados

\begin{tabular}{|c|c|c|}
\hline Programa & Principais características & Linhas de abordagem CS \\
\hline Metacognitive Training ${ }^{3}$ & $\begin{array}{l}\text { Programa de intervenção em grupo consistindo de oito sessões de } \\
\text { duração entre } 45 \text { e } 60 \text { minutos, cujo objetivo é conscientizar os pacientes } \\
\text { de distorções cognitivas sociais }\end{array}$ & $\begin{array}{l}\text { Estilos atribucionais disfuncionais e "jumping to conclusions" focando } \\
\text { situações sociais, processamento cognitivo "Teoria da Mente" (ToM) }\end{array}$ \\
\hline Emotion and ToM Imitation Training ${ }^{6}$ & $\begin{array}{l}\text { Programa de intervenção em grupo com duração de } 12 \text { semanas e } \\
\text { desenhado para esquizofrênicos }\end{array}$ & ToM e imitação de expressões faciais \\
\hline $\begin{array}{l}\text { Reabilitação cognitiva com ênfase } \\
\text { em informação social }{ }^{8}\end{array}$ & $\begin{array}{l}\text { Programa de intervenção individual consistindo de } 12 \text { sessões semanais } \\
\text { de } 40 \text { minutos }\end{array}$ & $\begin{array}{l}\text { "Social knowledge" (esquemas ou macroestruturas cognitivas a respeito } \\
\text { de como compreender comportamentos humanos) }\end{array}$ \\
\hline $\begin{array}{l}\text { Cognitive Enhancement Therapy } \\
\text { (CET })^{9,12,13,15}\end{array}$ & $\begin{array}{l}\text { Programa de intervenção em grupo para remediação de déficits } \\
\text { cognitivos e sociocognitivos para pacientes esquizofrênicos e } \\
\text { esquizoafetivos, cujo subprograma CS caracteriza-se por } 45 \text { sessões de } \\
\text { grupo de treino sociocognitivo }\end{array}$ & $\begin{array}{l}\text { Além de um subprograma neurocognitivo, disponibiliza um } \\
\text { subprograma CS cuja finalidade é estimular } 0 \text { aprendizado de } \\
\text { informações a respeito do ambiente social e estimular interações sociais }\end{array}$ \\
\hline $\begin{array}{l}\text { Social Cognitive Training } \\
\text { Program }(\mathrm{SCTP})^{10}\end{array}$ & $\begin{array}{l}0 \text { programa estudado neste trabalho consiste de intervenção em grupo, } \\
\text { realizado duas vezes por semana, em } 20 \text { sessões }\end{array}$ & $\begin{array}{l}\text { Reconhecimento de emoções (módulo do programa desenhado pelos } \\
\text { autores) e percepção social (módulo extraído do programa Terapia } \\
\text { Integrada da Esquizofrenia) }\end{array}$ \\
\hline $\begin{array}{l}\text { Social Cognition and Interaction } \\
\text { Training (SCIT) } 2,11,19\end{array}$ & $\begin{array}{l}\text { Programa de intervenção em grupo com duração de } 18 \text { a } 24 \\
\text { semanas, com ênfase em processos sociocognitivos disfuncionais em } \\
\text { esquizofrênicos }\end{array}$ & Percepção e discriminação de emoções faciais e ToM \\
\hline $\begin{array}{l}\text { Social Cognitive Enhancement Training } \\
(\text { SCET) })^{14}\end{array}$ & $\begin{array}{l}\text { Programa de intervenção em grupo, consistindo de duas sessões } \\
\text { semanais e duração de seis meses }\end{array}$ & $\begin{array}{l}\text { Percepção de pistas sociais em cartoons e organização deles na ordem da } \\
\text { informação contextual, além de discussão dos resultados }\end{array}$ \\
\hline Training of Affect Recognition (TAR) ${ }^{17,18}$ & $\begin{array}{l}\text { Programa de intervenção em grupo, consistindo de } 12 \text { sessões a ser } \\
\text { desenvolvido em duas sessões de } 45 \text { minutos por semana }\end{array}$ & Reconhecimento do afeto facial \\
\hline Cognitive Emotional Rehabilitation ${ }^{20}$ & $\begin{array}{l}\text { Programa de intervenção em grupo, com sessões semanais durando de } \\
75 \text { a } 90 \text { minutos e duração de seis meses }\end{array}$ & Percepção de emoções, ToM \\
\hline Instrumental Enrichment Programme ${ }^{21}$ & $\begin{array}{l}\text { Programa de intervenção em grupo, com sessões semanais com duração } \\
\text { de até } 1 \text { hora, aplicado pelos autores durante } 22 \text { semanas }\end{array}$ & $\begin{array}{l}\text { Aquisição de consciência de déficits, de conceitos a respeito da } \\
\text { dificuldade de reconhecer emoções; estímulo a novos processos de } \\
\text { aprendizado social, compreensão da utilidade dos processos cognitivos } \\
\text { envolvidos nas interações sociais, modificação das percepções de si } \\
\text { mesmo e de terceiros }\end{array}$ \\
\hline
\end{tabular}


Quatro estudos descreveram seus programas de tratamento explicitamente como metacognitivos ${ }^{3,6,20,21}$.

Os tipos de desfecho avaliados abrangeram melhoras em medidas de psicopatologia, cognição geral, processamento ToM, reconhecimento de emoções ou do afeto facial, desempenho social, interpretação e percepção de situações sociais e número de incidentes agressivos. A tabela 3 resume os principais parâmetros psicopatológicos, cognitivos e sociocognitivos avaliados em cada estudo.

A maior parte dos estudos documentou melhora significativa de parâmetros CS e até mesmo de parâmetros cognitivos gerais e de sintomatologia nos grupos recebendo programas metacognitivos baseados em CS, exceto Aghotor et al. ${ }^{3}$ (que não demonstraram diferenças significativas entre grupos em medidas de psicopatologia ou de cognição), Choi e Kwon ${ }^{14}$ (que, embora com seu programa tenham en- contrado melhora em déficits neurocognitivos, não conseguiram demonstrar algum impacto dessas melhoras sobre o funcionamento social, bem como efeito sobre reconhecimento de emoções) e Penn e Combs $^{16}$ (os quais registraram melhora da capacidade de identificação, mas não da de discriminação do afeto facial).

Alguns estudos comparando programas com foco em CS com programas não CS demonstraram especificidade dos tratamentos $26,9,13,15,18-21$.

Poucos estudos preocuparam-se em estudar a manutenção dos resultados favoráveis de seus programas ao longo do tempo ${ }^{8,11}$ ou em avaliar a resposta diferencial à exposição de pacientes com doença recente ou tardia aos programas, ${ }^{9}$.

Os trabalhos aqui revisados fazem pouca ou nenhuma menção ao papel dos psicofármacos utilizados por suas populações nos resultados obtidos.

Tabela 3. Avaliação de psicopatologia, cognição geral e CS nos estudos incluídos

\begin{tabular}{|c|c|c|}
\hline Estudo & Avaliação da psicopatologia e/ou da cognição geral & Avaliação da cognição social \\
\hline Penn e Combs, $2000^{16}$ & $\mathrm{BPRS}^{37}$ & $\begin{array}{l}\text { Face Emotion Identification Task (FEIT) }{ }^{38} \text {; Face Emotion Discrimination Task } \\
(\mathrm{FEDT})^{38}\end{array}$ \\
\hline Hogarty et al., $2004^{15}$ & $\begin{array}{l}\text { Velocidade de processamento, habilidades visuoespaciais, memória verbal, } \\
\text { memória de trabalho, linguagem, flexibilidade cognitiva, velocidade }\end{array}$ & Social Cognition Profile 39 \\
\hline
\end{tabular}
psicmo trabalho, linguagem, flexibilidade cognitiva, velocidade depressão, autoestima, temores cotidianos e energia

Roncone et al., 2004 21 Fluência verbal e função executiva; Accertamento Disabilità (versão italiana da Disability Assessment Schedule $)^{40}$

Wölwer et al., 2005 $\quad$ Atenção, memória de trabalho, compreensão situacional, funções executivas, fluência verbal; PANSS ${ }^{42}$

Choi e Kwon, 2006 $\quad$ Organização e sequenciamento perceptual, distinção entre detalhes essenciais e não essenciais aferidos pela versão coreana da Escala de Inteligência de Wechsler para Crianças Revisada ${ }^{45}$

Combs et al., 2007 $\quad$ PANSS ${ }^{19}$; WRAT-III reading ${ }^{48}$; Zigler-Glick Pre-morbid Social Competence Scale ${ }^{49}$; medidas de flexibilidade cognitiva

Eack et al., 2007 $\quad$ QI na baseline

Combs et al., 200911

Social Functioning Scale ${ }^{55}$

Eack et al., $2009^{9}$

Velocidade de processamento, memória verbal, memória de trabalho, habilidades linguísticas, velocidade psicomotora, estilo cognitivo; medidas de ajustamento em domínios social e vocacional e ajustamento em papéis sociais maiores e de sintomas positivos e negativos, ansiedade, depressão e autoestima

Gil Sanz et al., 2009 $\quad$ PANSS ${ }^{42}$; subteste Direct Digits do Integrative Program of Neuropsychological Exploration of the Test Barcelona ${ }^{60}$; Disability Scale of the World Health Organization $^{61}$

Matsui et al., $2009^{8}$

Funcionamento executivo, memória verbal, flexibilidade cognitiva, planejamento, capacidade estratégica e memória de trabalho

Aghotor et al., $2010^{3} \quad$ PANSS ${ }^{42}$

Mazza et al., $2010^{6}$

Versão italiana da BPRS 37,65 ; Personal and Social Performance Scal ${ }^{66}$; medidas de QI, memória de trabalho, velocidade psicomotora, memória verbal, função executiva e fluência verbal

Veltro et al., $2011^{20}$

QI, memória de trabalho, velocidade psicomotora, memória verbal, função executiva e fluência verbal

Fromann et al., $2003^{17}$

PANSS 42

Hogarty et al., $2006^{12}$

Major Role Adjustment Inventory ${ }^{70}$; Global Assessment Scale ${ }^{71}$; medidas de velocidade de processamento, inteligência e fluência verbal

Roberts e Penn, $2009^{2}$

Histórias ToM lidas aos sujeitos experimentais; reconhecimento de emoções por meio de cartoons; Mach IV Scale $e^{41}$

Performance of Facial Affect (PFA Test) ${ }^{43}$; Benton Face Recognition Test (BFRT) ${ }^{44}$

Social Behavior Sequencing Task ${ }^{46}$; Emotion Recognition Task ${ }^{47}$

Face Emotion Identification Test ${ }^{50}$; Face Emotion Discrimination Test $t^{50}$; Social Perception Scale ${ }^{51}$; Hinting Task ${ }^{52}$; Ambiguous Intentions Attributional Questionnaire ${ }^{53}$; Need for Closure Scale ${ }^{54}$; Social Functioning Scale ${ }^{55}$ The Mayer-Salovey-Caruso Emotional Intelligence Test (MSCEIT) ${ }^{56}$ Face Emotion Identification Test ${ }^{50}$; The Bell-Lysaker Emotion Recognition Task $(\text { BLERT })^{57}$; The Social Skill Performance Assessment (SSPA) ${ }^{58}$

Cognitive Style and Social Cognition Elegibility Interview and the Cognitive Styles Inventory ${ }^{59}$; The Mayer-Salovey-Caruso Emotional Intelligence Test (MSCEIT) ${ }^{56}$

Teste computadorizado de reconhecimento de emoções composto de 30 fotos coloridas selecionadas do NimStim Face Stimulus Set; Escala de Percepción Social ${ }^{62}$

Script Test ${ }^{63}$

Trecho do BADE procedure ${ }^{64}$, apropriado para avaliação do jumping to conclusions.

Advanced Theory of Mind Scale ${ }^{67}$; histórias contendo falsas crenças; questionários de Empatia ${ }^{68}$; Emotion Attribution Task Colleagues ${ }^{69}$

Advanced Theory of Mind Scale ${ }^{67}$; histórias contendo falsas crenças; Emotion Attribution Task Colleagues ${ }^{69}$

Performance of Facial Affect (PFA) ${ }^{43}$

Cognitive Style and Social Cognition Elegibility Interview and the Cognitive Styles Inventory ${ }^{59}$; medidas de autoconfiança, percepção social, capacidade suportiva e tolerância do Social Cognition Profile ${ }^{59}$

The Face Emotion Identification Task (FEIT) ${ }^{38}$; The Bell-Lysaker Emotion Recognition Task (BLERT) ${ }^{57}$; Hinting Task ${ }^{52 ;}$ The Awareness of Social Inference Test (TASIT)72; The Ambiguous Intentions Hostility Questionnaire - Ambiguous Items (AlHQ-A) ${ }^{53}$ 


\section{DISCUSSÃO}

A já consolidada ideia de que o tratamento psiquiátrico deve incluir tanto a recuperação sintomática quanto a funcional tem estimulado a pesquisa por estratégias terapêuticas não farmacológicas que visem à minimização dos efeitos dos transtornos mentais sobre as relações sociais dos pacientes. Prejuízos no funcionamento social provocam diminuição na qualidade de vida e aumento do risco de recaídas na esquizofrenia ${ }^{25}$.

Baseado nisso, muitos programas psicoterápicos cujo objetivo principal é a reabilitação social foram desenvolvidos, alguns deles tendo como foco principal as alterações cognitivas gerais presentes e já bem documentadas na esquizofrenia. Apesar disso, a relação entre cognição, sintomas esquizofrênicos e desempenho social ainda não está bem esclarecida 4 .

O estudo da CS, além de ser um importante auxiliar na compreensão da origem dos sintomas esquizofrênicos, tem gerado novas estratégias de treinamento dirigido aos portadores desse transtorno, o que se reflete em um número crescente de publicações sobre o tema.

CS é um termo abrangente, referindo-se aos processos cognitivos relacionados à convivência com outras pessoas e englobando uma ampla gama de linhas de pesquisa em que as principais são o reconhecimento de emoções, a compreensão de situações sociais e as habilidades ToM. Grosso modo, todas essas linhas estudam os processos mentais a partir dos quais compreendemos os outros seres humanos, seja porque os concebemos como indivíduos que têm desejos, crenças e intenções, seja porque somos capazes de identificar estados emocionais nas outras pessoas, com as quais também estamos aptos a sincronizar emocionalmente.

Tais informações sobre os outros são fundamentais para se entender como formamos nossas representações mentais acerca de outras mentes.

Na medida em que incorporam tanto a observação dos processos emocionais a que os outros estão sujeitos (os quais são, inclusive, passíveis de serem simulados por quem os observa) quanto as atitudes proposicionais de terceiros (desejos, crenças e intenções), as representações mentais a respeito das mentes dos outros são a chave para a previsão de seu comportamento e, portanto, para relacionamentos interpessoais saudáveis.

Esse tipo de representação mental está comprometido na esquizofrenia ${ }^{26}$ e os trabalhos aqui revisados mostram que incluir pacientes esquizofrênicos em atividades que estimulem diversas formas de análise, reflexão e exposição a domínios cognitivos sociais pode auxiliá-los a melhorar seu rendimento na interpretação adequada de estímulos sociais, na medida em que podem contribuir para a remediação de suas representações deficitárias. Essas melhoras refletir-se-iam na diminuição da gravidade de sintomas positivos e negativos (principalmente afastamento social) e, possivelmen- te, as intercorrências com agressividade, além da facilitação à ressocialização.

Os principais domínios CS explorados pelos programas estudados são o reconhecimento e percepção de emoções, inferência de estados mentais (ToM), tomada de perspectiva em situações sociais e imitação de ações sociais (Tabela 3). A maior parte dos programas associa diferentes tarefas envolvendo esses domínios, o que auxilia nos resultados favoráveis em termos de melhora clínica e CS. De fato, a atomização da CS em domínios distintos facilita o estudo de cada um deles, contudo a CS caracteriza-se essencialmente pelo funcionamento sinérgico de todos os domínios que a caracterizam.

\section{Reconhecimento e percepção de emoções}

O ser humano é altamente visual e emprega um tempo considerável de seu cotidiano olhando e analisando faces humanas. De fato, rostos são fontes valiosas de informações indispensáveis no convívio social. O processamento da informação oriunda de faces humanas parece ser separável em níveis neurais distinto ${ }^{27}$ : enquanto uma análise visual básica seria realizada no córtex visual, a percepção de faces seria processada no giro occipital inferior, a percepção da identidade facial no giro fusiforme lateral e a percepção de variáveis sociais da face, como olhar e formato da boca - diferentes nas diversas emoções -, seria realizada no sulco temporal superior. Amígdala e córtex pré-frontal decodificariam adicionalmente as informações de todas essas regiões.

A interpretação errônea do afeto facial por esquizofrênicos é um possível mecanismo de produção de sintomas como delírios persecutórios ou afastamento social. Felizmente, o reconhecimento do afeto facial parece ser passível de melhora por meio de treino ${ }^{27}$.

A maioria dos programas envolvendo treino em reconhecimento de afeto em esquizofrênicos mostrou melhora significativa no reconhecimento de emoções em relação aos controles, sendo a única exceção o SCTP10, que, contudo, foi estudado em uma amostra pequena de sujeitos experimentais.

Mesmo levando em conta as diferenças qualitativas e quantitativas dos programas - e elas são importantes, na medida em que eles variam muito em conteúdo, tempo e rotinas de aplicação (Tabela 2) -, todos parecem ser úteis na melhora do reconhecimento de emoções e, talvez por isso, possam promover melhoras clínicas em sintomas positivos ou negativos, conforme mostraram alguns dos trabalhos revisa$\operatorname{dos}^{6,17,18,20,21}$

Todavia, duas situações devem ser levadas em conta ao se interpretarem esses resultados: a primeira, e mais geral, é que a natureza da relação melhora clínica-funcional e melhora cognitivo-social ainda é um assunto em aberto e merece mais estudos antes de uma conclusão definitiva. A segunda, mais especificamente relacionada aos programas discutidos nesta seção, é que nenhum deles concentrou- 
-se especificamente no treinamento de reconhecimento de emoções; pelo contrário, boa parte combinou tarefas de reconhecimento de emoções, de inferência de estados mentais e de imitação, o que pode ter aumentado o poder de identificação de afetos nos sujeitos experimentais.

A motivação para determinada tarefa parece também ter um impacto sobre o desempenho, e isso é bem ilustrado no trabalho de Penn e Combs ${ }^{16}$, que mostraram que o simples reforço monetário para o acerto pode melhorar a performance dos sujeitos experimentais em tarefas de identificação (mas não de discriminação) de afetos faciais.

\section{Inferência de estados mentais (ou habilidades ToM)}

A habilidade mental humana automática de se atribuírem estados mentais a terceiros tem a finalidade principal de compreensão e predição comportamental, facilitando o convívio social.

É importante destacar o papel da automaticidade e da espontaneidade dessa habilidade em humanos saudáveis. Elas recheiam nossas descrições do ambiente social com expressões relativas a estados mentais, como "querer", "ter a intenção de", "acreditar", "pensar" etc.

As habilidades ToM parecem ser dissociáveis em dois domínios distintos: o domínio ToM implícita (ou ToMi), que é a capacidade automática propriamente dita e que permite um processamento rápido das informações do ambiente social; e o domínio ToM explícita (ou ToMe), que é a capacidade de aprender as regras do jogo social, de forma a otimizar o convívio com outros seres humanos ${ }^{28}$ por meio da reflexão.

ToMi e ToMe parecem recrutar circuitos neurais distintos e, do ponto de vista da eficiência, a ToMe é mais lenta, não permitindo um processamento on-line da informação social.

Indivíduos que nascem com prejuízos na capacidade automática de inferir estados mentais de terceiros (ToMi), como alguns autistas e esquizofrênicos, estão sujeitos ao desenvolvimento de dificuldades na interação social ou isolamento social e de sintomas psicóticos ${ }^{29}$. Felizmente, eles podem compensar essa dificuldade por meio do aprendizado de regras sociais (desenvolvimento do domínio ToMe), embora isso envolva maiores empregos de esforço e tempo.

É possível que os resultados favoráveis apresentados por muitos dos programas revisados em relação aos parâmetros ToM devam-se, pelo menos em parte, à possibilidade de que o julgamento supervisionado de emoções e sua descrição, a imitação de emoções avaliadas e o estímulo da mudança de perspectiva em relação à avaliação de uma situação social, além do simples suporte psicoterapêutico oferecido pelos programas estudados, fomentem o desenvolvimento de circuitos ToMe, podendo resultar, entre outros fatores diretamente associados à melhora do contato interpessoal, em diminuição de incidentes agressivos, conforme mostrado em sujeitos expostos ao SCIT ${ }^{19}$.

\section{Tomada de perspectiva em situações sociais}

A avaliação do ambiente social pode estar comprometida na esquizofrenia graças a vieses cognitivos afetando tomadas de decisão. Por exemplo, esses pacientes tendem a coletar quantidade insuficiente de informação oriunda do ambiente social para chegar a uma conclusão (jumping to conclusions), além de frequentemente acusarem pessoas por eventos negativos - em vez de avaliarem a possibilidade de eles serem multicausais (apresentam um estilo atribucional peculiar) - e de apresentarem diminuição da clareza de memórias autobiográficas, nas quais confiam excessivamente (déficits em processos metamnêmicos) $)^{30}$.

Esses prejuízos afetam o que Matsui et al. ${ }^{8}$ chamam de "social knowledge", um repertório de esquemas cognitivos sobre como as pessoas deveriam se comportar em determinadas situações sociais. Esses autores demonstraram que a reabilitação cognitiva melhora domínios constituintes desses esquemas, como o julgamento e o sequenciamento de eventos sociais. Todavia, o grupo controle de Matsui et al. era constituído apenas de indivíduos em seguimento medicamentoso, assim recebendo quantidade proporcionalmente menor de atenção que aqueles expostos ao programa. De qualquer forma, a maioria dos estudos que continham em seus programas tarefas relacionadas à avaliação de situações sociais comparou seus programas com intervenções ativas de estruturação variável e demonstrou resultados favoráveis $23,3,9,11-15,19-21$.

\section{Imitação}

O principal modo de representação mental do ser humano moderno baseia-se em sua capacidade de simbolização. Todavia, a representação simbólica parece ter sido precedida por um modo ancestral de representações baseado na imitação ${ }^{31}$. De fato, existem evidências de uma cultura mimética (mas não simbólica) no Homo erectus, que já desenvolvia ferramentas e utensílios de complexidade variável. Uma cultura baseada na elaboração de objetos manufaturados precisa envolver mecanismos de rememoração motora de etapas complexas de sua confecção, além de métodos eficientes de propagação desse conhecimento entre os mais jovens, a fim de que a cultura seja preservada. Tais métodos basearam-se na imitação.

No entanto, no Homo sapiens o mimetismo ultrapassa os limites da manufatura de utensílios e adquire uma dimensão representacional sociocognitiva, na medida em que atos motores aprendidos por imitação passam a ter um significado semântico, por exemplo, quando levamos a mão ao rosto para expressar vergonha. Outros aspectos vestigiais da cultura mimética em humanos modernos envolvem o papel da imitação no aprendizado e na compreensão de outras mentes em crianças $^{32}$ e a função central da representação mimética na arte, principalmente pantomima e dança ${ }^{31}$. 
Os circuitos cerebrais relacionados à imitação envolvem o sulco temporal superior (STS), responsável pelo processamento visual de ações de terceiros, a parte rostral do lóbulo parietal inferior e a parte posterior do giro frontal anterior e córtex pré-motor ventral adjacente ${ }^{33}$, que criam uma representação motora da ação observada, a ser reenviada ao STS. O STS fará um pareamento entre a descrição visual da ação e de sua representação motora, criando um significado adicional para a ação observada. Esse significado adicional relaciona-se diretamente à capacidade de representar internamente as ações observadas e executadas por terceiros (e não exclusivamente sua decodificação visual) e parece ser importante tanto no desenvolvimento de representações mentais sobre os outros quanto em mecanismos de empatia, ambos comprometidos na esquizofrenia e em outros transtornos mentais ${ }^{34,35}$.

Mazza et al. ${ }^{6}$ e Penn e Combs ${ }^{16}$ demonstraram que o treino imitativo pode melhorar parâmetros cognitivos sociais em esquizofrênicos e, em consequência, não apenas melhorar o desempenho interpessoal desses indivíduos, mas aliviar sintomas positivos e negativos do transtorno. $O$ treino imitativo pareceu apurar a compreensão que os sujeitos experimentais tinham das outras pessoas, principalmente por meio do desenvolvimento da habilidade de reconhecer emoções em terceiros. É sabido que a imitação se manifesta precocemente em seres humanos: bebês com 1 a 3 semanas de idade já são capazes de imitar a protrusão da língua e a abertura de boca encenados por adultos com quem interagem. Meltzoff ${ }^{36}$ propõe que, ao imitarem gestos, bebês começam a modular comparativamente sua produção comportamental com a de outros humanos, detectando coincidências entre elas, em um processo denominado pareamento intermodal ativo (PIA). No PIA, movimentos percebidos e movimentos executados seriam registrados em um único sistema representacional, um passo inicial para a posterior compreensão de estados mentais de terceiros, propiciada pela possibilidade de conexão cognitiva entre o mundo visível dos outros e os estados internos do bebê. Assim se configuraria inicialmente a capacidade humana de compreender outras mentes e também de sincronizar emocionalmente com elas.

\section{CONCLUSÃO}

É possível concluir, a partir do estudo dos programas aqui revisados, que intervenções metacognitivas dirigidas a domínios cognitivos sociais podem auxiliar na recuperação clínica e funcional de esquizofrênicos. Contudo, algumas considerações merecem ser feitas sobre os achados dos trabalhos revisados.

Um questionamento importante diz respeito à correlação entre melhora clínica e melhora de parâmetros cogni- tivos sociais, isto é, qual seria o sentido dessa correlação: a melhora clínica acarretaria melhoras cognitivas ou a recuperação cognitiva é que estaria por trás da recuperação clínica? Evidentemente, melhoras clínicas podem suscitar melhoras cognitivas (neste caso, as alterações cognitivas de um dado transtorno deveriam ser consideradas estado-dependentes); no entanto, a observação de que déficits cognitivos sociais podem ocorrer em indivíduos vulneráveis à esquizofrenia e em parentes de portadores de esquizofrenia ${ }^{34}$, bem como em períodos intercríticos da doença, sugere que esses déficits podem ser traço-dependentes e, portanto, passíveis inclusive de intervenções preventivas. Pesquisas envolvendo a aplicação desses programas em indivíduos vulneráveis poderiam auxiliar a responder essas questões.

Outro ponto a ser discutido que diz respeito a um fator considerado importante em relação aos estudos de eficiência de programas psicoterapêuticos em geral se refere ao argumento de que a maior atenção dispensada por terapeutas e pesquisadores a pacientes expostos a esses programas pode estar diretamente vinculada às suas melhoras clínicas e cognitivas. Esse efeito pode ter sido mais evidente nos trabalhos que não comparam seus programas com outras intervenções ativas.

A comparação de programas com foco em CS com outros não orientados para CS pode ser útil para demonstrar a especificidade das intervenções. A possibilidade de que CS seja um domínio cognitivo especializado e distinto de outros domínios cognitivos gerais permanece em aberto, principalmente porque, apesar de processar um tipo particular de informação, o domínio CS recruta diversos circuitos cognitivos não sociais ${ }^{28}$.

Muitos programas aqui revisados utilizaram métodos de reabilitação baseados em capacidades humanas como a imitação e o reconhecimento e identificação de emoções. A maior compreensão a respeito da neurofisiologia e da neuropsicologia dessas habilidades e de sua relação com a construção da mente humana moderna poderá ser valiosa para a elaboração de programas de tratamento e de prevenção de transtornos mentais como a esquizofrenia.

Futuros estudos deverão se concentrar em responder alguns questionamentos ainda em aberto a respeito de programas metacognitivos com enfoque em CS para o tratamento da esquizofrenia, incluindo a sua segurança, a durabilidade em médio e longo prazo dos efeitos sobre cognição, funcionamento social e psicopatologia e as populações de pacientes que podem obter mais benefícios com a exposição a esses programas.

\section{CONTRIBUIÇÕES INDIVIDUAIS}

Hélio Tonelli - Elaboração da revisão, leitura dos manuscritos selecionados e redação do artigo.

Fernanda Liboni - Elaboração da revisão, leitura dos manuscritos selecionados e redação do artigo. 
Diego Augusto Nesi Cavicchioli - Revisão crítica do manuscrito final.

\section{CONFLITOS DE INTERESSE}

Drs. Tonelli, Liboni e Cavicchioli declaram não ter conflitos de interesses financeiros que possam ter enviesado o presente estudo, pois não receberam nenhum suporte financeiro governamental ou comercial para sua realização. O financiamento do trabalho envolveu recursos próprios dos autores.

\section{REFERÊNCIAS}

1. Newman LS. What is social cognition? Four basic approaches and their implications for schizophrenia research. In: Corrigan PW, Penn DL. Social cognition and schizophrenia. American Psychological Association; 2004.

2. Roberts DL, Penn DL. Social cognition and interaction training (SCIT) for outpatients with schizophrenia: a preliminary study. Psychiatry Res. 2009;166(2-3):141-7.

3. Aghotor J, Pfueller U, Moritz S, Weisbrod M, Roesch-Ely D. Metacognitive training for patients with schizophrenia (MCT): feasibility and preliminary evidence for its efficacy. J Behav Ther Exp Psychiatry. 2010;41:207-11.

4. Twamley EW, Jeste DV, Bellack AS. A review of cognitive training in schizophrenia. Schizophr Bull. 2003;29(2):359-82.

5. Flavell J. Metacognitive aspects of problem solving. In: Resnick $L B$ (Ed.). The nature of intelligence. Hisdale, N.J.: Erlbaum; 1976.

6. Mazza M, Lucci G, Pacitti F, Pino MC, Mariano M, Casachia M, et al. Could schizophrenic subjects improve their social cognition abilities only with observation and imitation of social situations? Neuropsychol Rehabil. 2010;20(5):675-703.

7. Grynszpan 0, Perbal S, Pelissolo A, Fossati P, Jouvent R, Dubal S, et al. Efficacy and specificity of computer assisted cognitive remediation in schizophrenia: a meta-analytical study. Psychol Med. 2011;41(1):163-73.

8. Matsui M, Arai H, Yonezawa M, Sumiyoshi T, Suzuki M, Kurachi M. The effects of cognitive rehabilitation on social knowledge in patients with schizophrenia. Appl Neuropsychol. 2009;16(3):158-64.

9. Eack SM, Greenwald DP, Hogarty SS, Cooley SJ, Dibarry AL, Montrose DM, et al. Cognitive enhancement therapy for early course schizophrenia: effects of a two-year randomized controlled trial. Psychiatr Serv. 2009;60(11):1468-76.

10. Gil Sanz D, Diego Lorenzo M, Bengochea Seco R, Arrieta Rodriguez M, Lastra Martinez I, Sánchez Calleja R, et al. Efficacy of a social cognition training program for schizophrenic patients: a pilot study. Span J Psychol. 2009;12(1):184-91.

11. Combs DR, Elerson K, Penn DL, Tiegreen JA, Nelson A, Ledet SN, et al. Stability and generalization of Social Cognition and Interaction Training (SCIT) for schizophrenia: six month follow-up results. Schizophr Res. 2009;112(1-3):196-7.

12. Hogarty GE, Greenwald DP, Eack SM. Durability and mechanism of effects of cognitive enhancement therapy. Psychiatr Serv. 2006;57(12):1751-7.

13. Eack SM, Hogarty GE, Greenwald DP, Hogarty, SS, Keshavan MS. Cognitive enhancement therapy improves emotional intelligence in early course schizophrenia: preliminary effects. Schizophr Res. 2007;89(1-3):308-11.

14. Choi KH, Kwon JH. Social cognition enhancement training for schizophrenia: a preliminary randomized controlled trial. Community Ment Health J. 2006;42(2):177-87.

15. Hogarty GE, Flesher S, Ulrich R, Carter M, Greenwald D, Pogue-Geile M, et al. Cognitive enhancement therapy for schizophrenia: effects of a 2-year randomized trial on cognition and behavior. Arch Gen Psychiatry. 2004;61(9):866-76.

16. Penn DL, Combs DL. Modification of affect perception deficits in schizophrenia. Schizophr Res. 2000;46:217-29.
17. Frommann N, Streit M, Wölwer W. Remediation of facial affect impairments in patients with schizophrenia: a new training program. Psychiatry Res. 2003;117:281-4.

18. Wölwer W, Frommann N, Halfman S, Piaszek A, Streit M, Gaebel W. Remediation of impairments in facial affect recognition in schizophrenia: efficacy and specificity of a new training program. Schizophr Res. 2005;80:295-303.

19. Combs DR, Adams SD, Penn DL, Roberts D, Tiegreen J, Stem P. Social Cognition and Interaction Training (SCIT) for inpatients with schizophrenia spectrum disorders: preliminary findings. Schizophr Res. 2007;91:112-6.

20. Veltro F, Mazza M, Vendittelli N, Alberti M, Casacchia M, Roncone R. A comparison of the effectiveness of problem solving training and of cognitive-emotional rehabilitation on neurocognition, social cognition and social functioning in people with schizophrenia. Clin Pract Epidemiol Ment Health. 2011;7:123-32.

21. Roncone R, Mazza M, Frangou I, De Risio A, Ussorio D, Tozzini C, et al. Rehabilitation of theory of mind deficit in schizophrenia: a pilot study of metacognitive strategies in group treatment. Neuropsychol Rehabil. 2004;14(4):421-35.

22. Silver H, Goodman C, Knoll G, Isakov V. Brief emotion training improves recognition of facial emotions in chronic schizophrenia. A pilot study. Psychiatry Res. 2004;128:147-54.

23. Ross K, Freeman D, Dunn G, Garety P. A randomized experimental investigation of reasoning training for people with delusions. Schizophr Bull. 2011;37(2):324-33.

24. Penn D, Roberts DL, Munt ED, Silverstein E, Jones N, Sheitman B. A pilot study of social cognition and interaction training (SCIT) for schizophrenia. Schizophr Res. 2005;357-9.

25. Hanson-Ohayon I, Kravetz S, Levy I, Roe D. Metacognitive and interpersonal interventions for persons with severe mental illness: theory and practice. Isr J Psychiatry Relat Sci. 2009:46:141-8.

26. Bosco FM, Bono A, Bara BG. Recognition and repair of communicative failures: the interaction between Theory of Mind and cognitive complexity in schizophrenic patients. J Commun Disord. 2012;45(3):181-97.

27. Marwick K, Hall J. Social cognition in schizophrenia: a review of face processing. Br Med Bull. 2008:88:43-58

28. Frith CD, Frith U. Mechanisms of social cognition. Annu Rev Psychol. 2012;63:287-313.

29. Frith C. The cognitive neuropsychiatry of schizophrenia. Hove, UK: Lawrence Erlbaum Associates; 1992.

30. Moritz S, Vitzthum F, Randjibar S, Veckenstedt, R, Woodward TS. Detecting and defusing cognitive traps: metacognitive intervention in schizophrenia. Curr Opin Psychiatry. 2010;23(6):561-9.

31. Donalds M. Origins of the modern mind: Three Stages in the Evolution of Culture and Cognition. Boston: Harvard University Press; 1993.

32. Gopnik A, Meltzoff AN, Kuhl PK. The scientist in the crib: what early learning tell us about the mind. New York: Harper Collins Publishers; 2001.

33. lacoboni M. Neurobiology of imitation. Curr Opin Neurobiol. 2009;9(6):661-5.

34. Tonelli HA, Alvarez CE, Silva AA. Esquizotipia, habilidades "Teoria da Mente" e vulnerabilidade à psicose: uma revisão sistemática. Rev Psiq Clín. 2009;36(6):229-39.

35. Tonelli H. Empatia no transtorno bipolar. Rev Psiq Clín. 2011;38(5):207-8.

36. Meltzoff AN. Origins of theory of mind, cognition and communication. J Commun Disord. 1999;32(4):251-69.

37. Mueser KT, Curran PJ, McHugo GJ. Factor structure of the Brief Psychiatric Rating Scale in schizophrenia. Psychol Assess. 1997;9:196-204.

38. Kerr SL, Neale JM. Emotion perception in schizophrenia: specific deficit or further evidence of generalized poor performance? J Abnorm Psychol. 1993;102:312-8.

39. Selman RL, Schultz LH. Making a friend in youth. Chicago, III: University of Chicago Press; 1990.

40. Morosini PL, Veltro F, Carreta A, Gaio R, Palomba, U, Ventra C. Adattamento dello strumento in lingua italiana. Riv Sper Freniatr. 1988:3:541-63.

41. Christie R, Geis FL (Ed.) Studies in Machiavellianism. New York: New York Academic Press; 1970.

42. Kay SR, Fiszbein A, Opler LA. The positive and negative syndrome scale (PANSS) for schizophrenia. Schizophr Bull. 1987;13:261-76.

43. Ekman P, Friesen WV. Pictures of facial affect. Palo Alto, California: Consulting Psychologists Press; 1977. 
44. Benton AL, Sivan AB, Hamisher KS, Vamey NR, Spreen 0. Contributions to neuropsychological assessment, a clinical manual. 2nd ed. New York: Oxford University Press; 1994.

45. Park KS. KEDI-WISC Manual. Seoul: Korean Educational Institute; 1991.

46. Kwon JH, Chun JS, Choi KH. Validation study of the social behavior sequencing task: the assessment of the social cognitive ability of schizophrenics. Korean I Clin Psychol. 2003;22:629-39.

47. Lee SJ. Development of an emotional awareness test consisting of problem solving tasks. Korean J Social Personal Psychol. 2002;15:65-86.

48. Wilkinson GS. Wide Range Achievement Test Administration Manual. 3rd ed. Washington: Wide Range; 1993.

49. Glick M, Zigler E, Zigler B. Developmental correlates of age of first hospitalization in nonschizophrenic psychiatric patients. J Nerv Ment Dis. 1985;173(11):677-84.

50. Kerr SL, Neale JM. Emotion perception in schizophrenia: specific deficit or further evidence of generalized poor performance? J Abnorm Psychology. 1993;102:312-8.

51. Garcia S, Fuentes I, Ruiz JC, Gallach E, Roder V. Application of the IPT in a Spanish sample: evaluation of the "social perception subprogramme". Int I Psychol Psychol Ther. 2003;3:299-310.

52. Corcoran R, Mercer G, Frith C. Schizophrenia, symptomatology and social inference: investigating "theory of mind" in people with schizophrenia. Schizophr Res. 1995;17:5-13.

53. Combs DR, Penn DL, Wicher M, Waldheter E. The Ambiguous Intention Hostility Questionnaire (AIHQ): a new measure for evaluating attributional biases in paranoia. Cogn Neuropsychiatry. 2007;12(2):128-43.

54. Webster DM, Kruglanski AW. Individual differences in need for cognitive closure. J Pers Soc Psychol. 1994;67:1049-62.

55. Birchwood M, Smith J, Cochrane R, Wetton S, Copestake S. The Social Functioning Scale: the development and validation of a new scale of social adjustment for use in family intervention programmes with schizophrenic patients. Br J Psychiatry. 1990;157:853-9.

56. Mayer JD, Salovey P, Caruso DR, Sitarenios G. Measuring emotional intelligence with the MSCEIT V2.0. Emotion. 2003;3(1):97-105.

57. Bell M, Bryson G, Lysaker P. Positive and negative affect recognition in schizophrenia: a comparison with substance abuse and normal control subjects. Psychiatry Res. 1997;73(1-2):73-82.

58. Patterson TL, Moscona S, McKibbin CL, Davidson K, Jeste DV. Social skills performance assessment among older patients with schizophrenia. Schizophr Res. 2001;48:351-60.
59. Hogarty GE, Greenwald DP. Cognitive Enhancement Therapy: The Training Manual. Pittsburgh: Western Psychiatric Institute and Clinic; 2006.

60. Peña-Casanova J. Programa integrado de exploración neuropsicológica. Barcelona: Masson; 1991.

61. World Health Organization. A procedure and schedule for assessment of disability in patients with severe psychiatric disorders. Disability Assessment Schedule DAS-II. Geneva; 1985.

62. Garcia S. Rehabilitación de enfermos mentales crónicos: entrenamiento en percepción social. Valencia, Spain: Universidad de Valencia; 2003.

63. Matsui M, Sumiyoshi T, Yuuki H, Kato K, Kurachi M. Impairment of event schema in patients with schizophrenia: examination of script for shopping at supermarket. Psychiatry Res. 2006;143:179-87.

64. Moritz S, Woodward TS. A generalized bias against disconfirmatory evidence in schizophrenia. Psychiatry Res. 2006;142:157-5.

65. Roncone R, Ventura J, Impallomeni M, Falloon IR, Morosini PL, Chiaravalle E, et al. Reliability of an Italian standardized and expanded Brief Psychiatric Rating Scale (BPRS 4.0) in raters with high versus low clinical experience. Acta Psychiatr Scand. 1999;100:229-36.

66. Morosini PL, Magliano L, Brambilla L, Ugolini S, Pioli R. Development, reliability and acceptability of a new version of the DSM-IV Social and Occupational Functioning Assessment Scale (SOFAS) to assess routine social functioning. Acta Psychiatr Scand. 2000;101:323-9.

67. Happe FG. An advanced test for theory of mind: understanding of story character's thoughts and feelings by able autistic, mentally handicapped, and normal children and adults. J Autism Dev Disord. 1994;24:129-54.

68. Baron-Cohen $S$, Wheelwright $S$. The empathy quotient: an investigation with adults with Asperger Syndrome or high functioning autism and normal sex differences. J Autism Dev Disord. 2004;34(2):163-75

69. Blair RJ, Cipolotti L. Impaired social response reversal. A case of 'acquired sociopathy'. Brain. 2000;123:1122-41

70. Hogarty GE, Goldberg SC, Schooler NR. Drug and sociotherapy in the aftercare of schizophrenic patients. III: adjustment of nonrelapsed patients. Arch Gen Psychiatry. 1974:31:609-18

71. Endicott J, Spitzer RL, Fleiss JL, Cohen J. The Global Assessment Scale: a procedure for measuring overall severity of psychiatric disturbance. Arch Gen Psychiatry. 1976;33:766-71.

72. McDonald S, Flanagan S, Rollins J, Kinch J. TASIT: a new clinical tool for assessing social perception after traumatic brain injury. J Head Trauma Rehabil. 2003;18:219-38. 